Herz 2011 · 36:287-289

DOI 10.1007/s00059-011-3478-8

Published online: 8 June 2011

(c) Urban \& Vogel 2011

\author{
B. Maisch \\ Klinik für Innere Medizin - Kardiologie, Philipps-Universität Marburg, Marburg
}

\title{
Cardiovascular disease in cancer patients... only the tip of the iceberg?
}

\section{Which malignancies originate from or affect the heart and pericardium directly?}

With the exception of atrial myxomas, primary tumors of the heart are extremely rare, whereas metastases to the heart and pericardium are more frequent. As a rule of thumb, approximately $75 \%$ of primary tumors are benign and $75 \%$ of these are atrial myxomas. Rhabdomyomas, fibromas, fibroelastomas, hemangiomas, pericardial cysts, lipomas, hamartomas, teratomas, paragangliomas, and pheochromocytomas of the heart account for the benign tumors of the heart. Primary malignant tumors are of sarcomatous origin such as myxo-, lipo-, angio-, fibro-, leiomyo-, osteo-, synovial, rhabdomyo-, and neurofibrosarcomas, or they are lymphomas. Their incidence is between $0.0017 \%$ and $0.028 \%$ in the series discussed in [4]. Myxomas are by far the most frequent. Their detection is often surprising and always a highlight for the echocardiographer. An atrial thrombus should be considered as a potential differential diagnosis in atrial fibrillation. Whereas atrial myxomas can undergo cardiac surgery, the outcome of cardiac sarcoma is lethal, with rare exceptions such as when cardiac transplant is attempted to save the life of the patient.

Benign and malignant primary pericardial tumors comprise a similar spectrum of pathologies with a comparable prognosis (• Tab. 1).

Metastatic pericardial disease is by far the most frequent neoplastic disorder affecting the heart $[6,7]$. The differential diagnosis of a pericardial effusion of unknown etiology can be difficult, whereby the differentiation of malignant from non-malignant pericardial effusion obviously has an immense prognostic impact. Malignant cells in a pericardial effusion should be determined by diagnostic cytological examination. However, pericardial fluid cytology, though specific, has a variable sensitivity. The role of tumor markers determined in the pericardial fluid and the peripheral blood is not well established. In their original contribution, Karatolios et al. discuss the diagnostic impact of the concentrations of the tumor markers carcinoembryonic antigen (CEA), carbohydrate antigen (CA) 19-9, CA 72-4, squamous cell carcinoma (SCC) antigen, and neuron-specific enolase (NSE) in malignant and non-malignant pericardial effusions. Their findings indicate that malignant pericardial effusions are associated with significantly higher pericardial concentrations of the tumor markers CEA, CA 72-4 and CA 19-9. The measurement of CA 72-4 levels in pericardial fluid offered the best diagnostic accuracy. Elevation of pericardial fluid CA 72-4 should rule in malignancy as a probable diagnosis even if the cytological analysis is not diagnostic.

\section{What are the pathophysiological mechanisms leading to cardiotoxicity in antitumor therapy?}

Cardiotoxicity has been known for decades to arise from cumulative doses of anthracycline therapy, which is still 
Tab. 1 Benign and primary malignant tumors of the pericardium [3]

\begin{tabular}{|c|c|}
\hline Benign & Malignant \\
\hline $\begin{array}{l}\text { Lymphangioma } \\
\text { (simple and cystic } \\
\text { multilocular) }\end{array}$ & $\begin{array}{l}\text { Diffuse and focal malig- } \\
\text { nant mesothelioma }\end{array}$ \\
\hline $\begin{array}{l}\text { Hemangioma } \\
\text { (simple and } \\
\text { cavernous) }\end{array}$ & Hemangiosarcoma \\
\hline Lipoma & $\begin{array}{l}\text { Hemangioendothelio- } \\
\text { sarcoma }\end{array}$ \\
\hline $\begin{array}{l}\text { Cystic intrapericardi- } \\
\text { al teratoma (benign } \\
\text { pericardial cysts) }\end{array}$ & $\begin{array}{l}\text { Hemangiopericytosar- } \\
\text { coma }\end{array}$ \\
\hline $\begin{array}{l}\text { Focal mesothelioma } \\
\text { (hyperplasia) }\end{array}$ & Fibrosarcoma \\
\hline Epicardial papilloma & Malignant teratoma \\
\hline Leiomyoma & Malignant Schwannoma \\
\hline Neurofibroma & Liposarcoma \\
\hline Aberrant thymoma & $\begin{array}{l}\text { Aberrant synoviosar- } \\
\text { coma }\end{array}$ \\
\hline
\end{tabular}

a major and indispensible cancer drug. The "Swiss cheese-like" appearance of destroyed cardiac tissue in histological specimens following exposure to increasing doses of doxorubicin can be explained nowadays not only by its principal toxic mechanisms involving iron and redox reactions, but also by abnormal protein processing, hyper-activated innate immune responses, the inhibition of neuregulin-1 (NRG1)/ErbB (HER) signalling, impaired progenitor cell renewal/cardiac repair, and decreased vasculogenesis. Yu Shi, Mark Moon, Steven Dawood, Bruce McManus, and Peter P. Liu from Toronto present this expanding pathogenic spectrum in detail. They point out that, although multiple mechanisms involved in doxorubicin cardiotoxicity have been studied, no clinically proven treatment has yet been successfully established for the prevention or treatment of doxorubicin cardiomyopathy. Previous attempts with the iron chelator dexrazoxane or with classic heart failure treatments including angiotensin converting enzyme (ACE) inhibitors and $\beta$-blockade as preventive strategies were only partially successful, if at all. Novel therapies such as anti-miR-146 or recombinant neuregulin-1 to increase cardiomyocyte resistance to toxicity are yet to be tested and confirmed in randomized trials.
C. A. Geisberg and D. J. Lenihan review the antitumor effect and cardiovascular side-effects of trastuzumab, a monoclonal antibody to the ErbB2 (Her2nue) receptor over-expressed in $\mathrm{Her}_{2}{ }^{+}$ breast cancer. Trastuzumab-related cardiotoxicity underlines the importance of ErbB2 signaling in the heart. Through the family of ErbB receptors, neuregulin (NRG-1), as an important stress-mediated paracrine growth factor, promotes cardioprotection by improved myocyte cell survival, proliferation, differentiation, hypertrophy, and angiogenesis. Pre-clinical animal and early-phase studies in man suggest that recombinant NRG-1 holds promise as a new therapy in the treatment of various forms of heart failure, not only in the prevention of trastuzumab-induced heart failure. This might therefore develop into a new strategy in heart failure therapy in general.

Radiation therapy has saved or prolonged the lives of many tumor patients. It is an integral part of modern treatment strategies in many types of cancer. However, we are also aware of its unwanted side effects: since the atomic bombs in Hiroshima and Nagasaki during the Second World War, we know that wholebody exposure to radiation can lead to cardiovascular damage even decades later. Until recently, the heart was considered as relatively radioresistant. The currently recommended tolerance dose for the heart was $40 \mathrm{~Gy}$ in fractions of $5 \times 2 \mathrm{~Gy} /$ week if the whole organ is irradiated and even up to $65 \mathrm{~Gy}$ in the case of partial exposure [1]. A. Wittig and R. Engenhardt-Cabillic point out that this is not the case nowadays, since epidemiological findings indicate that the heart is one of the most critical dose-limiting organs in radiotherapy [8]. In their review, Wittig and Engenhardt-Cabillic distinguish three groups of individuals experiencing exposure to radiation:

1) Individuals receiving low doses of radiation $(<1 \mathrm{~Gy})$. This group includes atomic bomb survivors in Japan, nuclear industry workers, and even inadequately protected medical personnel revealing an excess relative risk for death from heart disease, mostly infarction, of 0.17 (90\% $\mathrm{CI}=0.01-0.36)$ per Sievert.
2) Individuals receiving low to intermediate doses of radiation (cardiac dosage: $0.04-4.75 \mathrm{~Gy}$ ). This includes patients with scatter radiation after treatment with X-rays for ankylosing spondylitis. The E/O ratio was 1.25 .

3) Individuals receiving intermediate to high doses of radiation. Irradiated patients with breast cancer, lung cancer, esophageal cancer, thymoma, or Hodgkin's disease belong to this group. For breast cancer patients undergoing adjuvant radiotherapy, a significant excess mortality of 1.27 was noted.

A special section is also devoted to ultramodern particle therapy in their overview.

\section{How can we assess cardiotoxicity early?}

The assessment of myocytolysis preceding heart failure and reduced cardiac performance is the prerequisite for its early or even prophylactic treatment, not only in suspected myocardial infarction but also after the application of antitumor agents with cardiotoxic potential. The measurement of cardio-specific serum biomarkers of necrosis or apoptosis can precede systolic or diastolic heart failure, when measured at the right time. Increased serum levels of troponin I can identify patients with myocardial necrosis or apoptosis at risk for cardiotoxicity. Consequently, NtproBNP or other biomarkers of heart failure will indicate increased levels of stress to the heart. This scenario has been studied in several studies by Claudia Cardinale and Carlo Maria Cipolla, Milan. In their recent work and in their contribution to Herz, they demonstrate clearly that the early and even prophylactic use of angiotensin-converting enzyme inhibitors proved to be an effective preventive strategy in cancer patients treated with anticancer drugs.

The method of choice not only for the detection of primary or secondary cardiac tumors, metastatic myocardial, and metastatic pericardial disease, but also for the assessment of the functional compromise after the application of cardiotoxic drugs is color flow Doppler echocardiography. Jayant K. Raikhel- 
kar, Richard M. Steingart, and Carol L. Chen from the Sloan Kettering Institute in New York describe in their excellent comprehensive review important echocardiographic findings in patients with cardiac tumors, with cardiac metastases, and during treatment with cardiotoxic substances. Echocardiography detects atrial myxomas as a benign and angiosarcoma as a malignant primary tumor of the heart. It is instrumental in the assessment of cardiac amyloidosis. The authors identify the echocardiographic modalities for the detection and the follow-up of diastolic and systolic heart failure in cancer patients. Pericardial effusion can be found even years after successful radiotherapy of the thorax in Hodgkin's disease, breast and lung cancer, as well as damage to cardiac valves and the coronaries. The combination of tumor and thrombus formation reaching from the inferior vena cava to the right atrium is a characteristic feature found in renal cell carcinoma.

\section{How can we successfully prevent or treat cardiovascular disease in tumor patients?}

Since cardiovascular diseases and cancer are the leading causes of death in the Western world, both may be found either independently of or influencing one other. In their contribution, Noutsias and Maisch see emerging fields of close cooperation in the monitoring of cancer patients for the prevention, early diagnosis, and treatment of cardiovascular diseases. At present, cardiovascular treatment in cancer patients should follow the current treatment recommendations for patients without cancer, since little or no randomized data are available on cancer patients with coronary artery disease (CAD) or heart failure. In tumor patients with CAD with or without prior stenting and who require extracardiac surgery, aspirin should not be discontinued or substituted by subcutaneous or intravenous heparin.

Highlights of this issue include two case reports: Naib, Steingart, and Chen, New York, report for the first time on multivessel coronary vasospasm in a patient with hepatocellular carcinoma un- dergoing treatment with the tyrosinase inhibitor sorafenib. Maisch, Burchert, Moll, and Pankuweit report on a patient in whom pericardioscopy with targeted epicardial and pericardial biopsy enabled the distinction between neoplastic, radiogenic, or cardiotoxic pericardial effusion after effective treatment of the neoplastic process, including stem cell therapy. The assessment of a correct, etiologically-based diagnosis was the prerequisite for successful intrapericardial treatment $[6,7]$, which in this case was intrapericardial high-dose triamcinolone.

As can be seen in this issue of Herz, collaboration between cardiologists and oncologists is far-reaching. Risk factors such as cigarette smoking, diabetes, and advanced age are common in patients with atherosclerosis and cancer. Current cancer therapies cause "collateral damage" to the heart more often than previously anticipated in the "war against cancer". Not only classic anthracyclines but also many of the newer chemotherapeutic agents improve the primary outcome of antitumor treatment but limit long-term prognosis considerably after the successful treatment of cancer. What we know about cancer therapy and this type of collateral damage is insufficient-indeed, it is just the tip of the iceberg.

\section{Corresponding address}

\section{Prof. B. Maisch}

Department of Internal

Medicine and Cardiology,

University Hospital Gießen and

Marburg (UKGM GmbH) and Philipps

University Marburg

Baldingerstr., 35043 Marburg

maisch@staff.uni-marburg.de

\section{References}

1. Emami B, Lyman J, Brown A et al (1991) Tolerance of normal tissue to therapeutic irradiation. Int J Radiat Oncol Biol Phys 21:109-122

2. Hampton T (2010) Cancer therapy can be hard on the heart: researchers aim to explain-and avoid-cardiotoxicity. JAMA 303:1019-1020

3. Kanjuh V, Tatic V, Seferovic PM, Ristic AD (2000) Benign and primary malignant tumors of the pericardium: pathological overview. In: Seferovic PM, Spodick PM, Maisch B (eds) Pericardiology. Contemporary answers to continuing challenges. Elsevier Science, Belgrade, pp 27-41
4. Lam KY, Dickens P, Chan ACL (1993) Tumors of the heart - a 20-year experience with a review of 12485 consecutive autopsies. Arch Pathol Lab Med 117:1027-1031

5. Lenihan DJ, Cardinale D, Cipolla CM (2010) The compelling need for a cardiology and oncology partnership and the birth of the International CardiOncology Society. Prog Cardiovasc Dis 53:94-105

6. Maisch B, Ristic AD, Seferovic PM, Tsang TSM (2011) Interventional pericardiology: pericardiocentesis, pericardioscopy, pericardial biopsy, balloon pericardiotomy and intrapericardial therapy. Springer, Heidelberg

7. Maisch B, Ristic AD, Pankuweit S (2010) Evaluation and management of pericardial effusion in patients with neoplastic disease. Prog Cardiovasc Dis 53:157-164

8. Schultz-Hector S, Trott KR (2007) Radiation-induced cardiovascular disease: is there epidemiological evidence compatible with the radiobiological data? Int J Radiation Oncology Biol Phys 67:10-18 